\title{
Electrochemical Properties of Biopolymers in Strong Acid and Application in Displacement Sensor
}

\author{
Shih-Chen Shi ${ }^{*}$ \\ Department of Mechanical Engineering, National Cheng Kung University (NCKU), \\ No. 1 University Road, Tainan 70101, Taiwan \\ (Received April 3, 2018; accepted March 20, 2019)
}

Keywords: electrochemical property, biopolymer, acid, potentiodynamic polarization, EIS

The electrochemical properties of thin films of two derivatives of the biopolymer hydroxypropyl methylcellulose (HPMC), namely, hydroxypropyl methylcellulose phthalate (HPMCP) and hydroxypropyl methylcellulose acetate succinate (HPMCAS), in a strongly acidic environment were studied. The corrosion resistance of HPMC-derivative-coated high-speed steel (HSS) was evaluated using electrochemical impedance spectroscopic measurements and potentiodynamic polarization. The results of Nyquist plots and Tafel polarization showed that HPMCP and HPMCAS have different corrosion resistance properties. HPMCP helps form oxide compounds with the HSS surface, and HPMCAS synthesizes new compounds on the surface with water, thus increasing the corrosion resistance pathways. In this study, derivatives of biopolymers are used to create an ecologically friendly and anticorrosion coating to protect the surface of HSS and to provide an anticorrosion mechanism in a strong acid environment.

\section{Introduction}

In recent years, many researchers have begun to use high polymers as novel anticorrosion materials for effective cost reduction. ${ }^{(1,2)}$ In addition to their use as anticorrosive agents in a corrosive environment, the use of high polymers to form protective organic coatings on the surface of a metal is a popular approach. The most commonly used coating material is epoxy resin, but it is generally not used independently. Usually, it is used to form composites with other materials; alternatively, nanomaterials are added to it to obtain a more corrosion-resistant material. Adding aluminum nanoparticles ${ }^{(3)}$ to epoxy resins results in steel substrate corrosion owing to the formation of a mixed oxide of iron and aluminum. This increases the durability of corrosion resistance. Elemental analysis showed that a mixed oxide of iron and aluminum was formed between the coating and the substrate, which improved the corrosion resistance.

In terms of the application of graphene to corrosion-resistant coatings, graphene was added to epoxy resins with a super-hydrophobic surface design to improve the corrosion resistance. ${ }^{(4)}$ The epoxy resin/graphene nanocomposite was first prepared via physical blending;

*Corresponding author: e-mail: scshi@mail.ncku.edu.tw https://doi.org/10.18494/SAM.2019.2279 
subsequently, a super-hydrophobic surface was created by transferring the surface topography of taioba (Xanthosoma sagittifolium) leaves to the epoxy resin/graphene nanocomposite through the transfer printing technique. The super-hydrophobic surface showed a reduced contact area between the coating and the corrosive liquid, which could reduce the corrosion rate. The inside of the coating showed that the graphene sheets diverted the penetration pathway of corrosive factors. Consequently, they required a longer time to permeate the coating, thereby reducing the corrosion rate. Silane was added to epoxy resins to evaluate the corrosion resistance $^{(5)}$ and mechanical properties. ${ }^{(6)}$ The performances of two types of silane, namely, $\gamma$-aminopropyltriethoxysilane ( $\gamma$-APS) and 1,2-bis[triethoxysilyl] ethane (BTSE), were studied. Epoxy resin with silane exhibited better corrosion resistance, as the crosslink density of the polymer resin increased after the addition of silane, which strengthened the overall structure. However, optimal levels of incorporation exist for both types of silane; exceeding these levels can weaken the corrosion resistance and mechanical properties.

In most of the above examples, an epoxy-resin-based material was primarily used. Some research teams have attempted to use biodegradable high polymers as corrosion-resistant materials considering environmental protection and sustainable development. In a series of studies, cellulose was adopted for corrosion resistance, using materials such as hydroxypropyl methylcellulose (HPMC) and hydroxyethyl cellulose (HEC). ${ }^{(7,8)}$ These materials were added to corrosive solutions as anticorrosive agents. The components of these anticorrosive agents form a physisorption layer on the metal surface during immersion, which reduces the contact between the metal surface and the corrosive solution, thus increasing the corrosion resistance.

HPMC has many excellent properties. There are many references related to practical applications, such as nanocomposites,,${ }^{(9,10)}$ functional coatings, ${ }^{(11-17)}$ reinforced composites, ${ }^{(18-20)}$ self-healing films, ${ }^{(21,22)}$ anticorrosion materials, ${ }^{(23)}$ and bioapplicable materials. ${ }^{(24-26)}$ HPMC has a certain degree of solubility in water. In previous studies, HPMC was mostly used for corrosion resistance in the form of an anticorrosive agent. In other words, HPMC was added to solutions in a corrosive operating environment ${ }^{(5,6)}$ to reduce their corrosiveness. However, as an HPMC-based coating is expected to partially dissolve in water, its corrosion resistance is likely to be unsatisfactory. Moreover, to align with the goal of sustainable development, the replacement of solution-type anticorrosive treatment with a solid coating is a better alternative for the environment. Thus, in this study, two derivatives of HPMC, namely, hydroxypropyl methylcellulose phthalate (HPMCP) and hydroxypropyl methylcellulose acetate succinate (HPMCAS), which retain the excellent film-forming capability of HPMC, were selected. Being acid-resistant and insoluble in water, the resultant films are applicable to real-world environments. Apart from corrosion-resistant coatings, HPMC materials can be used as a displacement sensor owing to their $\mathrm{pH}$ sensitivity. The main purpose of this study was to investigate the corrosion-resistant behavior and mechanism of HPMC derivatives in an extreme environment, or more specifically, a strongly acidic solution, in order to satisfy the requirements of real-world applications. 


\section{Materials and Methods}

\subsection{HPMCAS and HPMCP film preparation}

The material specifications of HPMCP and HPMCAS in this experiment can be found in previous literature. ${ }^{(23)}$ First, $20 \mathrm{~mL}$ of pure water was mixed with $80 \mathrm{~mL}$ of ethanol $(95 \%$ concentration) to form a mixed solution. Subsequently, $10 \mathrm{~g}$ of a powder of HPMC derivatives (HPMCP and HPMCAS) was added to the mixed solution and stirred until the powder was completely dissolved. Subsequently, $600 \mu \mathrm{L}$ of the solution was drawn with a micropipette, dropped onto the substrate (SKH51), and allowed to stand for $12 \mathrm{~h}\left(25^{\circ} \mathrm{C}, \mathrm{RH} 30 \%\right)$. The above procedure was repeated three times to complete the coating process.

\subsection{Analysis of electrochemical properties}

The liquids providing the corrosive environment of $\mathrm{pH} 1$ were prepared using a $0.5 \mathrm{M}$ $\mathrm{NaCl}$ solution and $1 \mathrm{M} \mathrm{HCl}$ solution. The electrochemical characteristics of the coating were measured by electrochemical impedance spectroscopy (EIS, Jiehan, HIOKI 3533-05) and the potentiodynamic polarization method (Jiehan, ECW-5000).

\subsection{Raman spectroscopy and Fourier-transfer infrared spectroscopy (FTIR) analysis}

Raman spectroscopy (BWII-RAMaker, Andor, $532 \mathrm{~nm}$ laser) and FTIR (Thermo Nicolet NEXUS 470, Golden Valley, MN, USA, GMI) were used to evaluate whether new substances or bonds were formed between the coating and the substrate after corrosion. As Raman spectroscopy and FTIR analysis can only be applied to the surface, the coating and the highspeed steel (HSS) sample were separated before the experiment. The HSS sample was analyzed after separation.

\section{Results and Discussion}

\subsection{Potentiodynamic polarization analysis}

The effect of coating thickness on the corrosion resistance can be found in the literature. ${ }^{(21)}$ The coating thickness in this study was controlled at $600 \pm 20 \mu \mathrm{m}$. Figure 1 illustrates the polarization curves of the HPMCAS and HPMCP coatings in corrosive solutions $(\mathrm{pH}, 1)$. The lowest point on the curve indicates the corrosion potential $\left(E_{c o r r}\right)$, and the corrosion current $\left(I_{c o r r}\right)$ can be obtained by data fitting using the Tafel extrapolation method. $I_{\text {corr }}$ was treated as an indicator of the corrosion resistance, with a lower value of $I_{\text {corr }}$ indicating better corrosion resistance. The electrochemical corrosion measurements of HPMCAS and HPMCP are shown in Table 1. The Tafel plots for HPMCAS and HPMCP yield $E_{\text {corr }}=-231.9$ and $-478.4 \mathrm{mV}$, respectively, which are more positive than that of bare HSS, where $E_{\text {corr }}=-547.5 \mathrm{mV}$. The corrosion currents $\left(I_{\text {corr }}\right)$ of HPMCAS and HPMCP were 10.2 and $5.5 \mu \mathrm{A} / \mathrm{cm}^{2}$, respectively, 


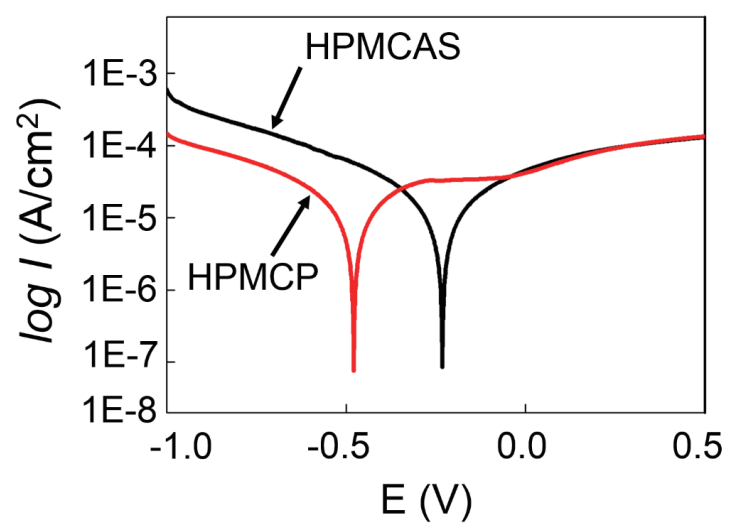

Fig. 1. (Color online) Polarization curves of HPMCAS and HPMCP coatings in $\mathrm{pH} 1$ solution.

Table 1

Fitting results obtained by Tafel extrapolation of polarization curves of HPMCP and HPMCAS coatings.

\begin{tabular}{lcccc}
\hline Material & $E_{\text {corr }}(\mathrm{mV})$ & $I_{\text {corr }}\left(\mu \mathrm{A} / \mathrm{cm}^{2}\right)$ & $\beta a(\mathrm{mV} / \mathrm{dec})$ & $\beta c(\mathrm{mV} / \mathrm{dec})$ \\
\hline HPMCAS & -231.9 & 10.2 & 239.84 & 225.55 \\
HPMCP & -478.4 & 5.5 & 133.75 & 114.96 \\
HSS & -547.5 & 26.3 & 65.4 & -159.9 \\
\hline
\end{tabular}

which are significantly lower than that of the HSS substrate $\left(26.3 \mu \mathrm{A} / \mathrm{cm}^{2}\right)$. Comparison of the fitting results of HPMCP and HPMCAS films shows that the $I_{\text {corr }}$ of HPMCP is relatively low, which shows that the HPMCP film provides better corrosion resistance than the HPMCAS films within a short soaking time. In order to further understand the effects of different soaking times on the properties of the coating, EIS research will be carried out.

\subsection{EIS analysis}

Figure 2 illustrates the EIS results for the HPMCAS and HPMCP coatings immersed in corrosive solutions $(\mathrm{pH}, 1)$ at different time points. As the corresponding Nyquist plots appear as semicircles in this figure, these data are determined by a time constant. The equivalent circuit model of the coating is shown in Fig. 3. In this figure, Rs is the solution resistance, which refers to the resistance of the corrosive solution between the working electrode and the auxiliary electrode. $R \_$film represents the resistance of the coating, and CPE_film represents the constant phase element (CPE), which is similar to the coating capacitance. Table 2 lists the results of fitting using the equivalent circuit model. The $R \_$film values of HPMCAS from 2 to $24 \mathrm{~h}$ were as follows: $39942,59813,73713,108130$, and $79782 \Omega$. The $R \_$film value was lowest at $2 \mathrm{~h}$ and gradually increased with immersion time. When the immersion time reached $16 \mathrm{~h}$, the $R \_$film value was the highest. However, the HPMCP coatings displayed a different behavior. The $R \_$film values of HPMCP from 2 to $24 \mathrm{~h}$ were as follows: 129090, 46399, 25395, 23290, and $29145 \Omega$. The $R$ film value was highest at $2 \mathrm{~h}$ and gradually decreased as the immersion time 


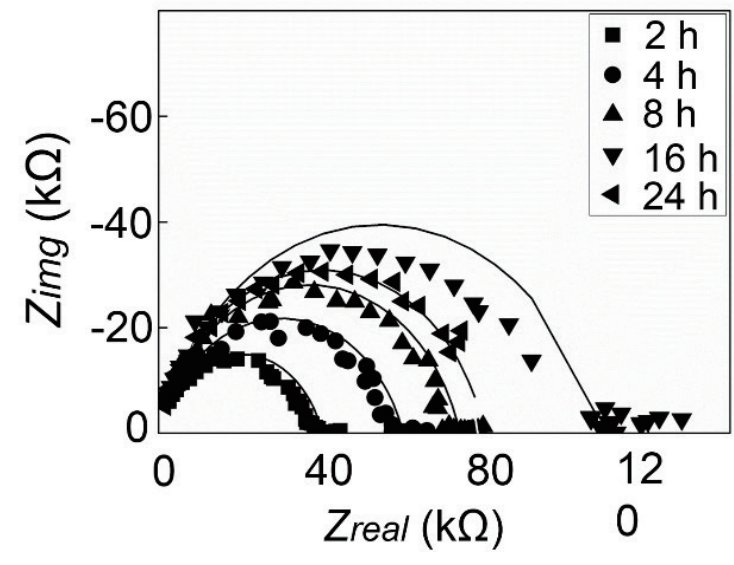

(a)

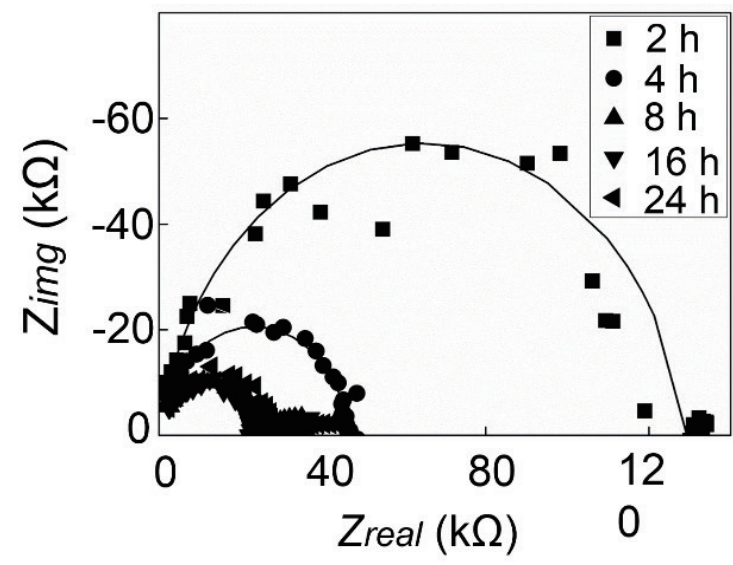

(b)

Fig. 2. EIS results for HPMCP and HPMCAS coatings after immersion in $\mathrm{pH} 1$ solutions for 2-24 h: (a) HPMCAS and (b) HPMCP. (Symbols are experimental data; lines are fitted results)

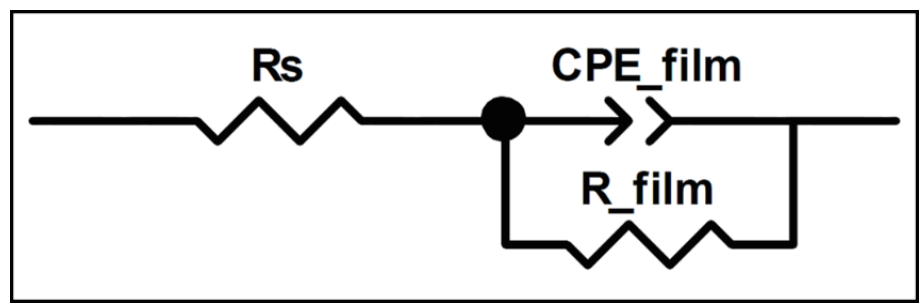

Fig. 3 Equivalent circuit model of HPMCP coating.

Table 2

Results of EIS data fitting using equivalent circuit model.

\begin{tabular}{lcccc}
\hline Material & Immersion time $(\mathrm{h})$ & $R \_$film $(\Omega)$ & $C P E \_$film $T$ & CPE_film_P \\
\hline \multirow{4}{*}{ HPMCAS } & 2 & 39942 & $1.4175 \times 10^{-9}$ & 0.8191 \\
& 4 & 59813 & $1.9194 \times 10^{-9}$ & 0.8004 \\
& 8 & 73713 & $1.3318 \times 10^{-9}$ & 0.8294 \\
& 16 & 108130 & $1.8274 \times 10^{-9}$ & 0.8031 \\
HPMCP & 24 & 79782 & $1.3664 \times 10^{-9}$ & 0.8394 \\
& 2 & 129090 & $3.7110 \times 10^{-10}$ & 0.9027 \\
& 4 & 46399 & $3.1651 \times 10^{-10}$ & 0.9242 \\
& 16 & 25395 & $7.3008 \times 10^{-10}$ & 0.8723 \\
& 24 & 23290 & $8.0130 \times 10^{-10}$ & 0.8709 \\
& & 29145 & $9.7943 \times 10^{-10}$ & 0.857 \\
\hline
\end{tabular}

increased. This result is consistent with the results for potentiodynamic polarization analysis. This indicates that, in an acidic environment, HPMCP has better corrosion resistance during the initial period. However, as the immersion time increases, its corrosion resistance declines rapidly. In contrast, HPMCAS has better corrosion resistance durability. 


\subsection{Volume fraction of water (VFW) analysis}

The volume fraction of water refers to the water volume within the coating, and it represents the extent of water intrusion from the environment into the coating and the formation of water channels. Hence, this measure is often used as an indicator of the corrosion resistance, where a higher VFW value is indicative of a weaker corrosion resistance. ${ }^{(27,28)}$ Equation (2) was used to analyze the VFW of the coatings via the EIS results.

$$
V F W=\frac{\log \left(C_{t} / C_{0}\right)}{\log 80}
$$

In Eq. (2), $C_{t}$ is the coating capacitance after time $t$ and $C_{0}$ is the coating capacitance at $t=0$. The " 80 " in the denominator refers to the dielectric constant of water. Because the previously described equivalent circuit model uses a CPE instead of capacitance $C$, the CPE must be converted into an equivalent capacitance ${ }^{(29-33)}$ using Eq. (3), and then substituted into Eq. (2).

$$
\left.C=R^{\left(\frac{1-C P E_{-} \text {film } P}{C P E_{-}}\right. \text {film_P }}\right) C P E_{-} f i l m_{-} T^{\left(\frac{1}{C P E_{-} \text {film }_{-} P}\right)}
$$

The results are shown in Fig. 4, where it can be observed that the VFW value increases with an increase in immersion time. This indicates that the intrusion of water from the environment into the coating increases over time. The results show that the HPMCAS film contains more water than the HPMCP film. To examine the effect of water on the subsequent corrosion resistance, we next analyze whether new substances or bonds have been generated between the coating and the HSS substrate.

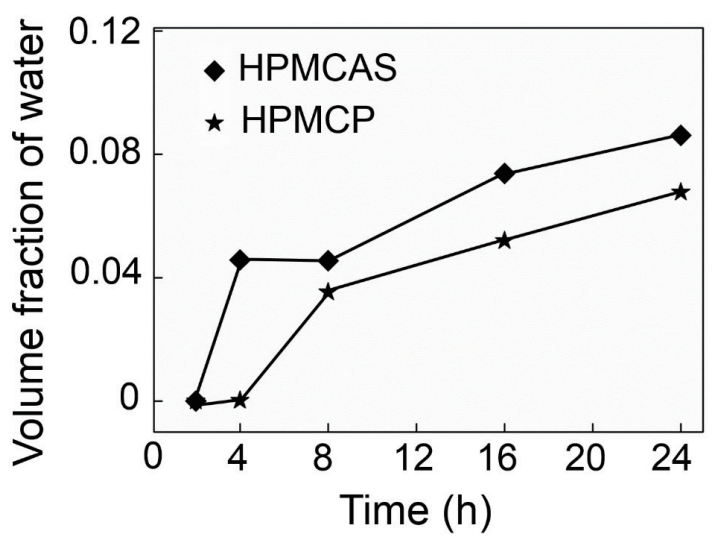

Fig. 4. Results of analysis of volume fraction of water for HPMCAS and HPMCP coatings. 


\subsection{Raman and FTIR analysis}

In this part of the experiment, uncorroded HSS, HPMCAS/HSS, and HPMCP/HSS samples were immersed in solution with $\mathrm{pH}=1$ for $24 \mathrm{~h}$. Raman spectroscopic analyses were used to determine whether new products had been generated between the coating and HSS substrate. The results are shown in Fig. 5(a). The 417 and $577 \mathrm{~cm}^{-1}$ peaks correspond to the characteristic peaks of iron oxides, ${ }^{(34,35)}$ which indicate that iron oxides were produced in the interface between the HPMCP coating and HSS. The EIS results reveal that after $24 \mathrm{~h}$ of reaction, $R \_$film exhibited the lowest value, indicating that severe corrosion had already occurred. However, no iron oxide signal was observed under the HPMCAS, which also indicated the absence of corrosion on the HSS surface. In previous studies, the formation of chromium oxides resulted in good corrosion protection. ${ }^{(36,37)}$ In Fig. 5(a), no chromium oxide was observed, thus excluding this possibility.

Previous studies revealed that HPMC performs as a corrosion inhibitor by adsorbing onto the substrate surface. ${ }^{(7)}$ Figure 5(b) shows the FTIR spectra of the HSS surface after HPMCAS/ HSS and HPMCP/HSS have been acid-soaked and the coating removed. No HPMC absorption was observed, thus excluding the possibility of HPMC absorption in both the HPMCAS and HPMCP coatings.

\subsection{Swelling effect analysis}

In previous studies, swelling of HPMCAS often occurred in various operating environments, increasing the thickness of the coating and transforming the coating into a gel layer. ${ }^{(22-24)}$ This increased the overall coating thickness. The thickness of the coatings in an environment with $\mathrm{pH} 1$ was measured in the experiment to identify any swelling, and the results are shown in Fig. 6. Figure 6(a) shows a schematic diagram of the measurement mainly for the changes in thickness after swelling (h). The measurement results shown in Fig. 6(b) indicate that the HPMCAS

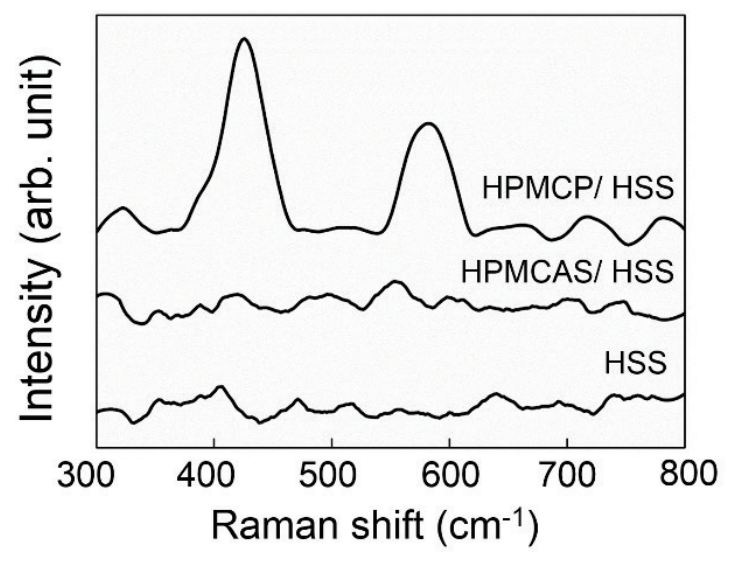

(a)

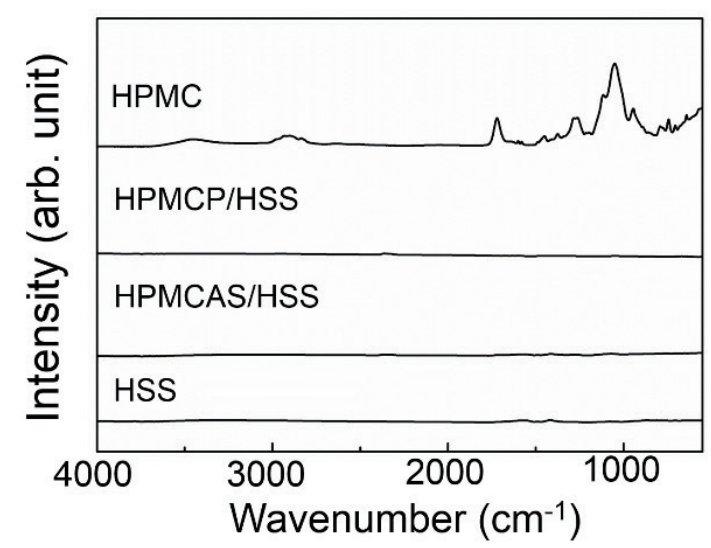

(b)

Fig. 5. (a) Raman and (b) FTIR results for the interface analysis between coatings and HSS. 


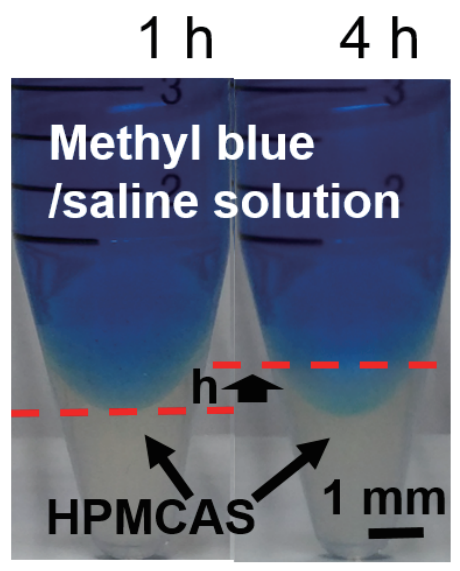

(a)

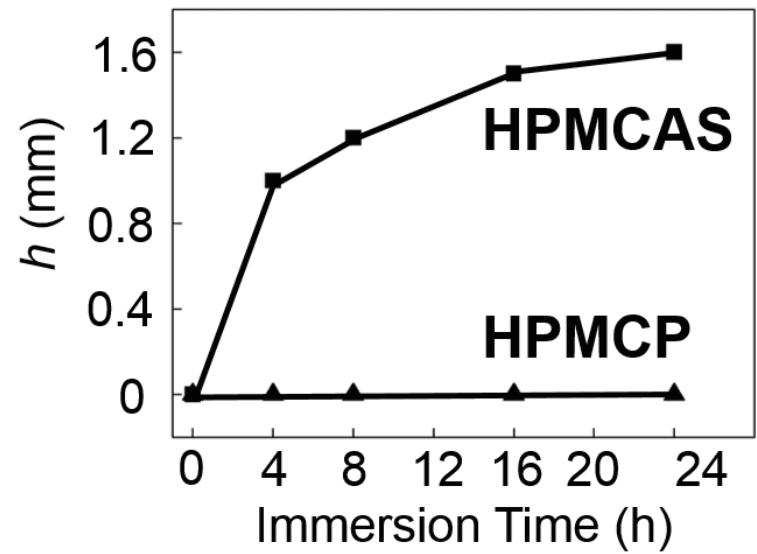

(b)

Fig. 6. (Color online) Swelling observation: (a) schematic diagram of swelling height measurement and (b) statistical results of swelling height measurement ( $20 \%$ margin of error).

coating exhibited significant swelling. It was observed that the thickness of the overall coating increased with immersion time. In contrast, the swelling effect was not significant in the HPMCP coating. It was speculated that this coating is more susceptible to dissolution in a strongly acidic environment. Therefore, the combined effects of swelling and dissolution resulted in insignificant changes in the coating thickness (within $24 \mathrm{~h}$ ).

Figure 6 illustrates that HPMCAS is prone to absorbing water, which leads to the swelling effect. This result agrees with the previous VFW experimental results. The HPMCAS coating absorbs water more easily than the HPMCP coating, and the water absorbed into the coating forms a gel layer with HPMCAS, providing a longer anticorrosion pathway. Consequently, the corrosion resistance of HPMCAS increases with time. However, as the coating becomes saturated with water, the gel layer begins to dissolve. Hence, the corrosion resistance declines over a long period of immersion. The HPMCP coating dissolves easily in a strongly acidic environment, and although it also has a swelling effect, longer anticorrosion pathways cannot be formed. In addition, the water absorbed into the HPMCP coating creates more aqueous water absorption pathways. Hence, the corrosion resistance is weakened significantly with time.

\section{Conclusions}

In this work, the electrochemical characteristics of biopolymers immersed in strongly acidic solution ( $\mathrm{pH}$ 1) were studied, and durability tests were also carried out. The conclusions of this study are as follows.

1. Compared with the HPMCP coating, the HPMCPAS coating has a longer corrosion resistance duration. Multilayered or sandwiched coatings can be used in practice to gain better corrosion resistance. 
2. Both HPMCP and HPMCAS absorb water in a strongly acidic environment. HPMCAS undergoes swelling, which increases the coating thickness and, consequently, the corrosion resistance. HPMCP undergoes both swelling and dissolution, which reduces the corrosion resistance of the coating.

3. HPMCAS absorbs water to form a gel layer within a certain period, which effectively protects the HSS substrate from corrosion. No oxide is observed on the HSS surface. In contrast, HPMCP creates more water pathways after water absorption, which causes corrosion on the surface of the HSS substrate.

4. HPMCAS has the potential to be used as a displacement sensor, as the coating thickness has a time-varying property.

\section{Acknowledgments}

The authors gratefully acknowledge the financial support for this project from the Ministry of Science and Technology in Taiwan (MOST 106-2221-E-006-092-MY3).

\section{References}

1 R. K. Farag and A. A. Farag: Cellulose-Based Hydrogels as Smart Corrosion Inhibitors: Cellulose-Based Superabsorbent Hydrogels (Springer Nature, Heidelberg, 2018) p. 1.

2 T. Gan, Y. Zhang, M. Yang, H. Hu, Z. Huang, Z. Feng, D. Chen, C. Chen, and J. Liang: Ind. Eng. Chem. Res. 57 (2018) 10786.

3 A. Madhankumar, S. Nagarajan, N. Rajendran, and T. Nishimura: J. Solid State Electrochem. 16 (2012) 2085.

4 K.-C. Chang, M.-H. Hsu, H.-I. Lu, M.-C. Lai, P.-J. Liu, C.-H. Hsu, W.-F. Ji, T.-L. Chuang, Y. Wei, and J.-M. Yeh: Carbon 66 (2014) 144.

5 M.-Y. Jiang, L.-K. Wu, J.-M. Hu, and J.-Q. Zhang: Corros. Sci. 92 (2015) 118.

6 M.-Y. Jiang, L.-K. Wu, J.-M. Hu, and J.-Q. Zhang: Corros. Sci. 92 (2015) 127.

7 J. F. Greene, J. W. Newman, K. C. Williamson, and B. D. Hammock: Chem. Res. Toxicol. 13 (2000) 217.

8 I. O. Arukalam: Carbohydr. Polym. 112 (2014) 291.

9 J. Sarkar, M. M. R. Mollick, D. Chattopadhyay, and K. Acharya: Bioprocess Biosystems Eng. 40 (2017) 351.

10 T. J. Pekin and N. J. Zvaifler: J. Clin. Invest. 43 (1964) 1372.

11 S.-C. Shi, J.-Y. Wu, and Y.-Q. Peng: Wear 408,409 (2018) 208.

12 S.-C. Shi and W.-K. Huang: Sens. Mater. 29 (2017) 1569.

13 S.-C. Shi and T.-F. Huang: Opt. Quantum Electron. 48 (2016) 532.

14 S.-C. Shi, J.-Y. Wu, and T.-F. Huang: Opt. Quantum Electron. 48 (2016) 474.

15 S.-C. Shi and T.-W. Chang: Opt. Quantum Electron. 50 (2018) 438.

16 S.-C. Shi and T.-W. Chang: Opt. Quantum Electron. 50 (2018) 440.

17 S.-C. Shi, T.-F. Huang, and J.-Y. Wu: Materials 8 (2015) 1738.

18 L. Chang, Z. Zhang, L. Ye, and K. Friedrich: Wear 262 (2007) 699.

19 S.-C. Shi and J.-Y. Wu: Sens. Mater. 29 (2017) 1491.

20 S.-C. Shi and J.-Y. Wu: Surf. Coat. Technol. 350 (2018) 1045.

21 S.-C. Shi and T.-F. Huang: Materials 10 (2017) 91.

22 E. A. Balazs, D. Watson, I. F. Duff, and S. Roseman: Arthritis \& Rheumatol. 10 (1967) 357.

23 S.-C. Shi and C.-C. Su: Materials 9 (2016) 612.

24 H. Kadry, T. A. Al-Hilal, A. Keshavarz, F. Alam, C. Xu, A. Joy, and F. Ahsan: Int. J. Pharm. 544 (2018) 285.

25 R. Zeeshan, Z. Mutahir, H. Iqbal, M. Ali, F. Iqbal, K. Ijaz, F. Sharif, A. T. Shah, A. A. Chaudhry, and M. Yar: Carbohydr. Polym. 193 (2018) 9.

26 S.-C. Shi and F.-I. Lu: Materials 9 (2016) 338.

27 S. Tambe, S. Singh, M. Patri, and D. Kumar: Prog. Org. Coat. 72 (2011) 315.

28 S. Haruyama, M. Asari, and T. Tsuru: Electrochemical Society (ECS) Proc. (1987) 197.

29 C. M. Gore, J. O. White, E. D. Wachsman, and V. Thangadurai: J. Mater. Chem. A 2 (2014) 2363. 
30 Q. Li and V. Thangadurai: J. Mater. Chem. 20 (2010) 7970.

31 Q. Li and V. Thangadurai: Fuel Cells 9 (2009) 684.

32 E. Chinarro, J. Jurado, F. Figueiredo, and J. Frade: Solid State Ionics 160 (2003) 161.

33 P. Hjalmarsson, M. Søgaard, and M. Mogensen: Solid State Ionics 180 (2009) 1395.

34 D. Phan, T. Häger, and W. Hofmeister: J. Raman Spectrosc. 48 (2017) 453.

35 M. Hanesch: Geophys. J. Int. 177 (2009) 941.

36 A. Ogwu, A. Oje, and J. Kavanagh: Mater. Res. Express 3 (2016) 045401.

37 A. F. Suzana, E. A. Ferreira, A. V. Benedetti, H. W. Carvalho, C. V. Santilli, and S. H. Pulcinelli: Surf. Coat. Technol. 299 (2016) 71.

\section{About the Author}

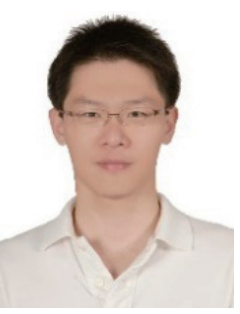

Shih-Chen Shi received his B.S. and M.S. degrees from National Cheng Kung University, Taiwan, in 1999 and 2001, respectively, and his Ph.D. degree from National Chiao Tung University, Taiwan, in 2005. From 2007 to 2012, he was the head of the R\&D Department at EVERLIGHT Electronic Co., Ltd., Taiwan. Since 2014, he has been an assistant professor at National Cheng Kung University. His research interests are in nanomaterials, tribology, LED applications, and sustainable materials. 